to appear in J. Phys. Soc. Jpn. 66, No. 5 (1997)

\title{
Fermi-liquid theory for a conductance through an interacting region attached to noninteracting leads
}

\author{
Akira Oguri \\ Department of Material Science, Faculty of Science, Osaka City University, \\ Sumiyoshi-ku, Osaka 558, Japan
}

(Received January 8, 1997)

\begin{abstract}
We study the conductance for the current through a finite interacting, possibly disordered region attached to two noninteracting leads within the Kubo formalism applying the perturbation theory for the local Fermi liquid developed by Yamada \& Yosida and by Shiba to this finite region. Assuming the validity of the perturbation expansion in the Coulomb interaction and the time reversal symmetry for the normal scattering potential in the finite region, we find that the contributions of the vertex correction to the dc conductance disappear at $T=0$ if the currents are measured in the leads. Consequently, the conductance is expressed as $g=\left(2 e^{2} / h\right)|t(0)|^{2}$. Here $t(0)$ is the transmission coefficient for single-particle-like excitation at the Fermi energy and is introduced by using the Green's function. The results are generalized to a quasi-one-dimensional system with a number of scattering channels.
\end{abstract}

PACS numbers: 72.10.-d, 72.10.Bg, 73.40.-c

Typeset using REVTEX 


\section{INTRODUCTION}

The effect of the Coulomb interaction on the process of resonant tunneling through a small region has been a subject of considerable investigation in the past few years. For studying the transport through such small systems theoretically, it is sometimes necessary to formulate the expression of the conductance so that both the Coulomb interaction and quantum mechanical interference effects can be treated exactly, and several attempts to obtain a rather general formulation have been made so far. 明 The purpose of this paper is to present one such attempt based on the Kubo formalism with the perturbation theory in the Coulomb interaction.

We consider a system in which the Coulomb interaction and the scattering potential due to the disorder are switched on only for the electrons staying in a finite region of an infinite system, and apply the perturbation analysis used in the microscopic version of the Fermi liquid theory for the impurity Anderson model 36 to the present system. We find that the dc conductance for zero-temperature $T=0$ is written in a Landauer-type form 6.8 if the assumptions shown in the following are satisfied. This result may be regarded as an

extension of the relation shown in the noninteracting case by Economou \& Soukoulis for one dimension and by Fisher \& Lee for higher dimensions. $9-11$ The assumptions to be made are

i) the Coulomb interaction and the normal spin-independent scattering potential are restricted for electrons in a finite spatial region, and two semi-infinite noninteracting leads are coupled to this region,

ii) perturbation expansion in the Coulomb interaction is valid,

iii) the single-particle-like excitation at the chemical potential $\mu$ does not decay, i.e., the imaginary part of the proper self-energy which is no longer diagonal in the wave-number indices is zero at $T=0$.

The assumption $i$ ) specifies the model, and means that the Hamiltonian does not contain a spin-flip-scattering due to the Kondo-type exchange interaction, the spin-orbit coupling, 
and so on. The noninteracting leads make the scattering problem of the present system well defined, and enable us to introduce the scattering coefficients making use of the asymptotic form of the single-particle Green's function in the leads. Furthermore, the presence of the semi-infinite leads seems to make the Fermi-liquid description for low-energy states, the wave-length of which is much longer than the size of the central region $L$, probable. Under the assumption $i$ ), we use the diagrammatic analysis for the perturbation in the Coulomb interaction considering the central region as an impurity with a finite volume, 3 目 and calculate the dc conductance by extracting the $\omega$-linear imaginary part of the currentcurrent correlation function following Shiba. by Langer \& Ambegaokar as a basic assumption in deriving the Friedel sum rule for interacting Fermi systems 12 As Langer \& Ambegaokar also mentioned, this assumption holds in the perturbation theory. It means that $i i i)$ is not an independent assumption and deduced from the two assumptions $i$ ) and $i i$ ). We will show this explicitly by calculating the imaginary part of the self-energy following Yamada \& Yosida. 3.

In this paper we consider mainly the system in one dimension. This is because the extension to higher dimensions is possible following along almost the same lines. We show the outline of the extension in the last part of this paper. Owing to the presence of the noninteracting leads, the perturbation theory will probably make sense for low-energy states with long enough wave-length even in the one-dimensional case. The Tomonaga-Luttingerliquid-like behavior seems to be valid for higher-energy region where the wave-length of a massless-boson-like excitation is shorter than the size of the central region L. 31,32 Recently, the value of the renormalization factor $K$ of the conductance in the Tomonaga-Luttinger liquid, $g=K e^{2} / h$ (per spin), was discussed by several authors 13 in relation to the experiment by Tarucha, Honda, and Saku.17 In the perturbation theory, the conductance can be expressed in a Landauer-type form, so that $g$ becomes $e^{2} / h$ (per spin) when a resonant tunneling occurs, i.e., in the case the transmission coefficient for the single-particle-like excitation at the Fermi energy is unity. This result itself seems to be consistent with the result obtained by Shimizu with the phenomenological description of a Fermi liquid in one 
dimension 16

We note that some of the ideas used in the present study were already applied to the

current through a single Anderson-Wolff impurity 2,19 in our previous work, 20 where the expression of the conductance was studied for the use of numerical calculations with the quantum Monte Carlo method.21,22 The expression for the conductance through the single impurity has also been studied precisely with the Keldysh formalism by Hersfield, Davies and Willkins.23 The conductance through a two-impurity Anderson-Wolff model has been formulated by Izumida, Sakai and Shimizu with a linear response theory for the use of the calculations with the numerical renormalization group method.24

In section [1], we introduce the transmission and reflection coefficients, and confirm that the coefficients for the single-particle-like excitation at the Fermi energy satisfy the unitarity condition. In section [II], we calculate the imaginary part of the proper self-energy with the perturbation theory in the Coulomb interaction, and show the assumption iii) is deduced from $i$ ) and $i i$ ). In section $\mathbb{I \nabla}$, we study the expression for the dc conductance $g$ within the Kubo formalism, and show that the contributions of the vertex correction to $g$ are zero at $T=0$ if the currents are measured in the noninteracting leads. Owing to this property, $g$ can be expressed in terms of the transmission coefficient. We also discuss the role the vertex correction with relation to the back flow. In section $\bar{\nabla}$, we generalize the results obtained for one dimension in Sec. $\amalg \sqrt{\mathbb{I V}}$ to the quasi-one-dimensional system which is finite along the transverse direction.

\section{SCATTERING COEFFICIENTS}

In this section, we introduce the transmission and reflection coefficients for a singleparticle-like excitation, and show that the coefficients for the excitation at the Fermi energy satisfy the unitarity condition if the assumption iii) holds.

We consider an one-dimensional system which consists of three parts: a finite central region at $0<x<L$, and two semi-infinite lead wires at $-\infty<x \leq 0$ and $L \leq x<+\infty$. 
The Hamiltonian is given by

$$
\begin{aligned}
\mathcal{H} & =\mathcal{H}_{0}+\mathcal{H}_{V}+\mathcal{H}_{U}, \\
\mathcal{H}_{0} & =\sum_{\sigma} \int_{-\infty}^{+\infty} d x \psi_{\sigma}^{\dagger}(x)\left(-\frac{1}{2 m} \frac{\partial^{2}}{\partial x^{2}}-\mu\right) \psi_{\sigma}(x), \\
\mathcal{H}_{V} & =\sum_{\sigma} \int_{0}^{L} d x \psi_{\sigma}^{\dagger}(x) V(x) \psi_{\sigma}(x), \\
\mathcal{H}_{U} & =\frac{1}{2} \sum_{\sigma \sigma^{\prime}} \int_{0}^{L} \int_{0}^{L} d x d x^{\prime} \psi_{\sigma}^{\dagger}(x) \psi_{\sigma^{\prime}}^{\dagger}\left(x^{\prime}\right) U\left(x, x^{\prime}\right) \psi_{\sigma^{\prime}}\left(x^{\prime}\right) \psi_{\sigma}(x),
\end{aligned}
$$

where $\psi_{\sigma}^{\dagger}(x)$ creates an electron with spin $\sigma$ at position $x$. The spin-independent scattering potential $V(x)$ and the Coulomb repulsion $U\left(x, x^{\prime}\right)$ are restricted for the electrons staying in the central region; and $U\left(x, x^{\prime}\right)=U\left(x^{\prime}, x\right)$. When the size of the central region $L$ is of the order of the Fermi wave-length, the model is reduced to an Anderson-Wolff impurity.1. 19 In this limit the perturbation theory is valid for all values of $U$. Our working hypothesis is that the ground state is changed continuously when the size $L$ is increased from an atomic size to larger one owing to the presence of the noninteracting leads. Although it is not evident that the hypothesis is valid for whole region of the parameter space of the Hamiltonian, which may be valid at least for a finite region in the parameter space where $U, V$, and $L$ are small enough. Unless, otherwise noted, we will be using units in which the Planck constant is $\hbar=1$.

We now consider the transmission and reflection coefficients for the system described by the Hamiltonian Eq. (11). For this purpose, we introduce the retarded Green's function

$$
G\left(x, x^{\prime} ; \omega+i 0^{+}\right)=-i \int_{0}^{\infty} d t\left\langle\left\{\psi_{\sigma}(x, t), \psi_{\sigma}^{\dagger}\left(x^{\prime}, 0\right)\right\}\right\rangle e^{i\left(\omega+i 0^{+}\right) t}
$$

where $\mathcal{O}(t) \equiv e^{i \mathcal{H} t} \mathcal{O} e^{-i \mathcal{H} t},\langle\cdots\rangle$ denotes the thermal average $\operatorname{Tr}\left[e^{-\beta \mathcal{H}} \cdots\right] / \operatorname{Tr} e^{-\beta \mathcal{H}}$ with $\beta$ being the inverse temperature $1 / T$, and the curly brackets denote the anticommutator. The spin index has been omitted from the left-hand side because the expectation value is assumed to be independent of whether spin is up or down. The Dyson equation for the Green's function is written, in the real space, as

$$
G\left(x, x^{\prime} ; z\right)=G_{0}\left(x, x^{\prime} ; z\right)+\int_{0}^{L} \int_{0}^{L} d x_{1} d x_{2} G_{0}\left(x, x_{1} ; z\right) \Sigma\left(x_{1}, x_{2} ; z\right) G\left(x_{2}, x^{\prime} ; z\right)
$$




$$
=G_{0}\left(x, x^{\prime} ; z\right)+\int_{0}^{L} \int_{0}^{L} d x_{1} d x_{2} G_{0}\left(x, x_{1} ; z\right) \mathcal{T}\left(x_{1}, x_{2} ; z\right) G_{0}\left(x_{2}, x^{\prime} ; z\right)
$$

where $G_{0}\left(x, x^{\prime} ; z\right)$ is the free Green's function corresponding to $\mathcal{H}_{0}$, and the region of the integral is taken to be $0 \leq x_{1}, x_{2} \leq L$ because the Coulomb interaction and the scattering potential are restricted for electrons in the central region. The proper self-energy $\Sigma\left(x_{1}, x_{2} ; z\right)$ and the scattering matrix $\mathcal{T}\left(x_{1}, x_{2} ; z\right)$ contain both of the effects due to $\mathcal{H}_{U}$ and $\mathcal{H}_{V}$. Note that the functions introduced here are symmetric with respect to the permutations of $x$ and $x^{\prime}$ because of the time reversal symmetry of $\mathcal{H}$

$$
\left\{\begin{array}{l}
G\left(x, x^{\prime} ; z\right)=G\left(x^{\prime}, x ; z\right) \\
\Sigma\left(x, x^{\prime} ; z\right)=\Sigma\left(x^{\prime}, x ; z\right) \\
\mathcal{T}\left(x, x^{\prime} ; z\right)=\mathcal{T}\left(x^{\prime}, x ; z\right)
\end{array} .\right.
$$

Therefore, the discontinuity of the Green's function at the real axis in the complex $z$-plane corresponds to the imaginary part

$$
\left[G\left(x, x^{\prime} ; \varepsilon+i 0^{+}\right)-G\left(x, x^{\prime} ; \varepsilon-i 0^{+}\right)\right] / 2 i=\operatorname{Im} G\left(x, x^{\prime} ; \varepsilon+i 0^{+}\right)
$$

and the similar relation holds also for $\Sigma\left(x, x^{\prime} ; z\right)$ and $\mathcal{T}\left(x, x^{\prime} ; z\right)$. Specifically, the free retarded Green's function is obtained, for $\omega>-\mu$, as

$$
G_{0}\left(x, x^{\prime} ; \omega+i 0^{+}\right)=-i \pi \rho(\omega) e^{i k\left|x-x^{\prime}\right|},
$$

where $k \equiv \sqrt{2 m(\omega+\mu)}$, and $\rho(\omega) \equiv m / \pi k$ is the density of states.

When both $x$ and $x^{\prime}$ belong to the leads, the asymptotic form of the full Green's function can be written explicitly, making use of Eqs. (1) and (77):

$$
G\left(x, x^{\prime} ; \xi_{k}+i 0^{+}\right)=e^{i k x}\left[1-i \pi \rho\left(\xi_{k}\right) \mathcal{T}_{k k}\left(\xi_{k}+i 0^{+}\right)\right]\left\{-i \pi \rho\left(\xi_{k}\right)\right\} e^{-i k x^{\prime}}
$$

for $x^{\prime}<0$ and $L<x$;

$$
G\left(x, x^{\prime} ; \xi_{k}+i 0^{+}\right)=\left[e^{i k x}-i \pi \rho\left(\xi_{k}\right) \mathcal{T}_{-k k}\left(\xi_{k}+i 0^{+}\right) e^{-i k x}\right]\left\{-i \pi \rho\left(\xi_{k}\right)\right\} e^{-i k x^{\prime}}
$$

for $x^{\prime}<x<0$. Here $\mathcal{T}_{k^{\prime} k}(z)$ is the Fourier transform of $\mathcal{T}\left(x, x^{\prime} ; z\right)$ 


$$
\mathcal{T}_{k^{\prime} k}\left(\omega+i 0^{+}\right)=\int_{0}^{L} \int_{0}^{L} d x_{1} d x_{2} e^{-i k^{\prime} x_{1}} \mathcal{T}\left(x_{1}, x_{2} ; \omega+i 0^{+}\right) e^{i k x_{2}},
$$

and the frequency $\omega$ is taken to be an on-shell value $\omega=\xi_{k}\left[\equiv k^{2} / 2 m-\mu\right]$ in Eqs. (8) and (9). Physically, the retarded Green's function $G\left(x, x^{\prime} ; \xi_{k}+i 0^{+}\right)$represents a propagation of a single-particle-like excitation; an additional electron for $\xi_{k}>0$, or a hole for $-\mu<\xi_{k}<0$. When $\xi_{k}>0$, for instance, the right-hand side of Eq. (8) is relating to a probability amplitude for the process in which an electron created at $x^{\prime}<0$ in the left lead is transmitted to the right lead with $L<x$. Similarly, Eq. (9) represents the process in which an electron created at $x^{\prime}<0$ propagates as an incident wave $e^{i k x}$ or a reflected wave $e^{-i k x}$ for $x^{\prime}<x<0$. Therefore it seems natural to define the transmission and reflection coefficients using Eqs. (8) and (9);

$$
\left\{\begin{array}{l}
t\left(\xi_{k}\right) \equiv 1-i \pi \rho\left(\xi_{k}\right) \mathcal{T}_{k k}\left(\xi_{k}+i 0^{+}\right) \\
r\left(\xi_{k}\right) \equiv-i \pi \rho\left(\xi_{k}\right) \mathcal{T}_{-k k}\left(\xi_{k}+i 0^{+}\right)
\end{array} .\right.
$$

With this definition, the unitarity condition $\left|t\left(\xi_{k}\right)\right|^{2}+\left|r\left(\xi_{k}\right)\right|^{2}=1$ is expressed in the form of an optical theorem

$$
\operatorname{Im} \mathcal{T}_{k k}\left(\xi_{k}+i 0^{+}\right)=-\frac{\pi \rho\left(\xi_{k}\right)}{2}\left[\left|\mathcal{T}_{k k}\left(\xi_{k}+i 0^{+}\right)\right|^{2}+\left|\mathcal{T}_{-k k}\left(\xi_{k}+i 0^{+}\right)\right|^{2}\right]
$$

In the non-interacting system, the coefficients defined by Eq. (11) coincide with the scattering coefficients which are obtained from the asymptotic form of the wave function, and thus Eq. (12) holds automatically. However, when the Coulomb interaction is switched on, it is not obvious whether Eq. (12) holds or not. We show in the following that the unitarity condition Eq. (12) holds at $T=0$ for the single-particle-like excitation at Fermi energy $\xi_{k}=0$ if the assumption iii) mentioned in the Introduction holds for any positions $x$ and $x^{\prime}$ in the central region as

$$
\operatorname{Im} \Sigma\left(x, x^{\prime} ; i 0^{+}\right)=0
$$

For simplicity, we temporarily write the integral equation Eq. (3) in the operator form, $\boldsymbol{G}=\boldsymbol{G}_{\mathbf{0}}+\boldsymbol{G}_{\mathbf{0}} \boldsymbol{\Sigma} \boldsymbol{G}$, and take the frequency for the operators to be $z=i 0^{+}$. In this form the $\mathcal{T}$-matrix is written as 


$$
\mathcal{T}=\Sigma+\Sigma G_{0} \Sigma+\Sigma G_{0} \Sigma G_{0} \Sigma+\cdots
$$

Since Eq. (13) means that $\boldsymbol{\Sigma}$ is Hermitian at $T=0$, i.e. $\boldsymbol{\Sigma}=\boldsymbol{\Sigma}^{\dagger}$, the optical theorem can be derived following essentially the same way with that in the noninteracting case making use of an operator identity 2 国

$$
\begin{aligned}
\mathcal{T}-\mathcal{T}^{\dagger} & =\left(\Sigma+\Sigma G_{0}^{\dagger} \Sigma+\cdots\right)\left(G_{0}-G_{0}^{\dagger}\right)\left(\Sigma+\Sigma G_{0} \Sigma+\cdots\right) \\
& =\mathcal{T}^{\dagger}\left(G_{0}-G_{0}^{\dagger}\right) \mathcal{T}
\end{aligned}
$$

A diagonal element in the wave-number indices is written as

$$
\mathcal{T}_{k k}\left(i 0^{+}\right)-\mathcal{T}_{k k}^{*}\left(i 0^{+}\right)=-\int_{-\infty}^{+\infty} \frac{d k^{\prime}}{2 \pi} \mathcal{T}_{k^{\prime} k}^{*}\left(i 0^{+}\right) 2 \pi i \delta\left(\xi_{k^{\prime}}\right) \mathcal{T}_{k^{\prime} k}\left(i 0^{+}\right)
$$

where $k$ is the Fermi wave number which satisfies $\xi_{k}=0$. Since the contribution of the integral in the right-hand side of Eq. (16) comes only from $k^{\prime}= \pm k$, the optical theorem Eq. (12) holds for the single-particle-like excitation at $\xi_{k}=0$.

As already mentioned, the assumption Eq. (13) was introduced by Langer and Ambegaokar to derive the Friedel sum rule for interacting Fermi systems: ${ }^{2}$ it is expressed in the present case as

$$
\begin{aligned}
\Delta n & \equiv-\frac{1}{\pi} \int_{-\infty}^{0} d \omega \int_{-\infty}^{+\infty} d x \operatorname{Im}\left[G\left(x, x ; \omega+i 0^{+}\right)-G_{0}\left(x, x ; \omega+i 0^{+}\right)\right] \\
& =\frac{1}{2 \pi i} \operatorname{Tr} \log \left[\boldsymbol{I}+\left(\boldsymbol{G}_{\mathbf{0}}-\boldsymbol{G}_{\mathbf{0}}^{\dagger}\right) \mathcal{T}\right]
\end{aligned}
$$

where the frequency for the operators in the second line is taken to be $z=i 0^{+}$. We note that Eq. (13) can be derived with the perturbation theory in the Coulomb interaction and the calculation is performed in the next section.

For the later convenience, we introduce here the Matsubara Green's function

$$
G\left(x, x^{\prime} ; i \varepsilon_{n}\right)=-\int_{0}^{\beta} d \tau\left\langle T_{\tau} \psi_{\sigma}^{M}(x, \tau) \psi_{\sigma}^{\dagger M}\left(x^{\prime}, 0\right)\right\rangle e^{i \varepsilon_{n} \tau},
$$

where $\mathcal{O}^{M}(\tau) \equiv e^{\tau \mathcal{H}} \mathcal{O} e^{-\tau \mathcal{H}}$, and $\varepsilon_{n}=(2 n+1) \pi / \beta$. The Dyson equation for the Matsubara Green's function is obtained by setting $z=i \varepsilon_{n}$ in Eqs. (3) and (团). The free Matsubara Green's function is obtained as 


$$
\begin{aligned}
G_{0}\left(x, x^{\prime} ; i \varepsilon\right) & =m \frac{e^{i \kappa(i \varepsilon)\left|x-x^{\prime}\right|}}{i \kappa(i \varepsilon)}, \\
\kappa(i \varepsilon) & \equiv \sqrt{2 m}\left[\mu^{2}+\varepsilon^{2}\right]^{1 / 4} e^{i \varphi(i \varepsilon) / 2} \operatorname{sgn} \varepsilon, \\
\varphi(i \varepsilon) & \equiv \arg (\mu+i \varepsilon) .
\end{aligned}
$$

where $\operatorname{sgn} \varepsilon$ is the sign function. The range of the value of phase $\varphi(i \varepsilon)$ is defined as $-\pi<$ $\varphi \leq \pi$. Thus in the limit $\varepsilon \rightarrow 0^{ \pm} ; \varphi\left(i 0^{ \pm}\right)=0$ for $\mu>0$, whereas $\varphi\left(i 0^{ \pm}\right)= \pm \pi$ for $\mu<0$. Now we note some characteristic features in the limit $\varepsilon \rightarrow 0^{+}$. When $\mu>0, G_{0}\left(x, x^{\prime} ; i 0^{+}\right)$ is an oscillatory function of $\left|x-x^{\prime}\right|$, and the singular contribution with the sign function in the right-hand side of Eq. (19) corresponds to the imaginary part of the retarded function for $\omega=0$, i.e. $\operatorname{Im} G_{0}\left(x, x^{\prime} ; i 0^{+}\right)$, owing to the symmetry property Eqs. (5) and (6). On the other hand, when $\mu<0, G_{0}\left(x, x^{\prime} ; i 0^{+}\right)$is a real and exponentially decreasing function of $\left|x-x^{\prime}\right|$, and the singular term disappears from the right-hand side of Eq. (19) because $\exp \left[i \varphi\left(i 0^{ \pm}\right) / 2\right] \operatorname{sgn} 0^{ \pm}=i$

\section{IMAGINARY PART OF THE SELF-ENERGY}

In this section, we calculate the imaginary part of the proper self-energy up to the order $\varepsilon^{2}$ with the perturbation theory in the Coulomb interaction. 12627 We show that the imaginary part is proportional to $\varepsilon^{2}$ at $T=0$, and thus Eq. (13) holds in the perturbation theory.

As already mentioned, the self-energy satisfies the relation corresponding to Eq. (6),

$$
\operatorname{Im} \Sigma\left(x, x^{\prime} ; \varepsilon+i 0^{+}\right)=\left[\Sigma\left(x, x^{\prime} ; \varepsilon+i 0^{+}\right)-\Sigma\left(x, x^{\prime} ; \varepsilon-i 0^{+}\right)\right] / 2 i
$$

owing to the symmetry property Eq. (5). It means that the imaginary part of the retarded self-energy $\Sigma\left(x, x^{\prime} ; \varepsilon+i 0^{+}\right)$is related to the $\operatorname{singular} \operatorname{sgn} \varepsilon_{n}$ contributions in the Matsubara function $\Sigma\left(x, x^{\prime} ; i \varepsilon_{n}\right)$ by the analytic continuation, and this correspondence holds for any $x$ and $x^{\prime}$. Therefore we can obtain the $\varepsilon^{2}$-term following the calculations in the case of the usual Fermi liquidente27 in which the self-energy is a diagonal quantity. Yamada showed

precisely that the singular contribution up to the order $\varepsilon_{n}^{2}$ comes only from diagrams which 
contain three intermediate Green's functions.425 Fig. 11 shows the skeleton diagram for the simplest example, and the expression for the imaginary part is written after the analytic continuation as 26

$$
\begin{aligned}
& \operatorname{Im} \Sigma^{(2)}\left(x, x^{\prime} ; \varepsilon+i 0^{+}\right) \\
& =\int_{-\infty}^{+\infty} \frac{d \varepsilon^{\prime} d \varepsilon^{\prime \prime}}{(2 \pi)^{2}}\left[\operatorname{coth} \frac{\varepsilon^{\prime}-\varepsilon}{2 T}-\tanh \frac{\varepsilon^{\prime}}{2 T}\right]\left[\tanh \frac{\varepsilon^{\prime \prime}}{2 T}-\tanh \frac{\varepsilon^{\prime \prime}+\varepsilon-\varepsilon^{\prime}}{2 T}\right] \\
& \quad \times \int_{0}^{L} \int_{0}^{L} d y d y^{\prime} U\left(x^{\prime}, y^{\prime}\right) U(x, y) \operatorname{Im} G\left(y^{\prime}, y ; \varepsilon^{\prime \prime}+i 0^{+}\right) \\
& \quad \times\left\{2 \operatorname{Im} G\left(x, x^{\prime} ; \varepsilon^{\prime}+i 0^{+}\right) \operatorname{Im} G\left(y, y^{\prime} ; \varepsilon+\varepsilon^{\prime \prime}-\varepsilon^{\prime}+i 0^{+}\right)\right. \\
& \left.\quad-\operatorname{Im} G\left(y, x^{\prime} ; \varepsilon^{\prime}+i 0^{+}\right) \operatorname{Im} G\left(x, y^{\prime} ; \varepsilon+\varepsilon^{\prime \prime}-\varepsilon^{\prime}+i 0^{+}\right)\right\} .
\end{aligned}
$$

Here $G$ is the full Green's function which contains all the effects of the scattering potential and the Coulomb interaction. At $T=0$, the hyperbolic functions in Eq. (23) are replaced by the sign functions as $\left[\operatorname{sgn}\left(\varepsilon^{\prime}-\varepsilon\right)-\operatorname{sgn}\left(\varepsilon^{\prime}\right)\right]\left[\operatorname{sgn}\left(\varepsilon^{\prime \prime}\right)-\operatorname{sgn}\left(\varepsilon^{\prime \prime}+\varepsilon-\varepsilon^{\prime}\right)\right]$. Thus the region of the integral for the frequencies are reduced to $\int_{0}^{\varepsilon} d \varepsilon^{\prime} \int_{\varepsilon^{\prime}-\varepsilon}^{0} d \varepsilon^{\prime \prime}$, and the area of the integral region becomes $\varepsilon^{2} / 2$. Therefore the expression for small $\varepsilon$ is obtained setting the frequencies for the Green's functions in Eq. (23) to be zero;

$$
\begin{aligned}
& \operatorname{Im} \Sigma^{(2)}\left(x, x^{\prime} ; \varepsilon+i 0^{+}\right) \\
& \simeq \frac{\varepsilon^{2}}{2 \pi^{2}} \int_{0}^{L} \int_{0}^{L} d y d y^{\prime} U\left(x^{\prime}, y^{\prime}\right) U(x, y) \operatorname{Im} G\left(y^{\prime}, y ; i 0^{+}\right) \\
& \quad \times\left\{2 \operatorname{Im} G\left(x, x^{\prime} ; i 0^{+}\right) \operatorname{Im} G\left(y, y^{\prime} ; i 0^{+}\right)-\operatorname{Im} G\left(y, x^{\prime} ; i 0^{+}\right) \operatorname{Im} G\left(x, y^{\prime} ; i 0^{+}\right)\right\} .
\end{aligned}
$$

The imaginary part is proportional to $\varepsilon^{2}$ for any $x$ and $x^{\prime}$ because the $\varepsilon^{2}$-dependence is coming from the hyperbolic functions. It is convenient for proceeding next step to rewrite Eq. (24) by introducing the bare vertecies $\gamma_{\uparrow \downarrow}$ and $\gamma_{\uparrow \uparrow}$ [see Fig. 2]

$$
\begin{aligned}
& \operatorname{Im} \Sigma^{(2)}\left(x, x^{\prime} ; \varepsilon+i 0^{+}\right) \\
& \simeq \frac{\varepsilon^{2}}{2 \pi^{2}} \int_{0}^{L} \cdots \int_{0}^{L} \prod_{i=1}^{6} d y_{i} \operatorname{Im} G\left(y_{6}, y_{5} ; i 0^{+}\right) \operatorname{Im} G\left(y_{1}, y_{2} ; i 0^{+}\right) \operatorname{Im} G\left(y_{3}, y_{4} ; i 0^{+}\right) \\
& \quad \times\left[\gamma_{\uparrow \downarrow}\left(x, y_{5} ; y_{3}, y_{1}\right) \gamma_{\uparrow \downarrow}\left(y_{2}, y_{4} ; y_{6}, x^{\prime}\right)+\frac{1}{2} \gamma_{\uparrow \uparrow}\left(x, y_{5} ; y_{3}, y_{1}\right) \gamma_{\uparrow \uparrow}\left(y_{2}, y_{4} ; y_{6}, x^{\prime}\right)\right],
\end{aligned}
$$

where 


$$
\begin{aligned}
& \gamma_{\uparrow \downarrow}\left(x_{4}, x_{3} ; x_{2}, x_{1}\right) \equiv U\left(x_{1}, x_{2}\right) \delta\left(x_{1}-x_{4}\right) \delta\left(x_{2}-x_{3}\right) \\
& \gamma_{\uparrow \uparrow}\left(x_{4}, x_{3} ; x_{2}, x_{1}\right) \equiv U\left(x_{1}, x_{2}\right)\left[\delta\left(x_{1}-x_{4}\right) \delta\left(x_{2}-x_{3}\right)-\delta\left(x_{1}-x_{3}\right) \delta\left(x_{2}-x_{4}\right)\right]
\end{aligned}
$$

We now include the higher order terms following Yamada, 127 which can be completed just by replacing the bare vertices $\gamma_{\sigma \sigma^{\prime}}$ with the full ones $\Gamma_{\sigma \sigma^{\prime}}$ [see Fig. [3]

$$
\Gamma_{\sigma \sigma^{\prime}}\left(x_{4}, x_{3} ; x_{2}, x_{1}\right) \equiv \Gamma_{\sigma \sigma^{\prime} ; \sigma^{\prime} \sigma}\left(x_{4}, x_{3} ; x_{2}, x_{1}: 0,0 ; 0,0\right)
$$

This is because the singular $\varepsilon_{n}^{2} \operatorname{sgn} \varepsilon_{n}$ contributions in the Matsubara function $\Sigma\left(x, x^{\prime} ; i \varepsilon_{n}\right)$ can be obtained by summing up all the diagrams with the three intermediate Green's functions. Therefore, we can write down the expression which is exact up to $\varepsilon^{2}$-term at $T=0$ by symmetrizing Eq. (25) with respect to the interchange of $x$ and $x^{\prime}$ making use of Eq. (5), then adding the equivalent expression in which the set of variables for the integration $\left\{y_{5}, y_{3}, y_{1}\right\}$ and $\left\{y_{6}, y_{4}, y_{2}\right\}$ are interchanged, and finally replacing the bare vertices by the full ones;

$$
\begin{aligned}
& \operatorname{Im} \Sigma\left(x, x^{\prime} ; \varepsilon+i 0^{+}\right) \\
& \simeq \frac{\varepsilon^{2}}{8 \pi^{2}} \int_{0}^{L} \cdots \int_{0}^{L} \prod_{i=1}^{6} d y_{i} \operatorname{Im} G\left(y_{6}, y_{5} ; i 0^{+}\right) \operatorname{Im} G\left(y_{1}, y_{2} ; i 0^{+}\right) \operatorname{Im} G\left(y_{3}, y_{4} ; i 0^{+}\right) \\
& \times\left[\Gamma_{\uparrow \downarrow}\left(x^{\prime}, y_{6} ; y_{4}, y_{2}\right) \Gamma_{\uparrow \downarrow}\left(y_{1}, y_{3} ; y_{5}, x\right)+\Gamma_{\uparrow \downarrow}\left(x, y_{5} ; y_{3}, y_{1}\right) \Gamma_{\uparrow \downarrow}\left(y_{2}, y_{4} ; y_{6}, x^{\prime}\right)\right. \\
& \quad+\Gamma_{\uparrow \downarrow}\left(x^{\prime}, y_{5} ; y_{3}, y_{1}\right) \Gamma_{\uparrow \downarrow}\left(y_{2}, y_{4} ; y_{6}, x\right)+\Gamma_{\uparrow \downarrow}\left(x, y_{6} ; y_{4}, y_{2}\right) \Gamma_{\uparrow \downarrow}\left(y_{1}, y_{3} ; y_{5}, x^{\prime}\right) \\
& \quad+\frac{1}{2} \Gamma_{\uparrow \uparrow}\left(x^{\prime}, y_{6} ; y_{4}, y_{2}\right) \Gamma_{\uparrow \uparrow}\left(y_{1}, y_{3} ; y_{5}, x\right)+\frac{1}{2} \Gamma_{\uparrow \uparrow}\left(x, y_{5} ; y_{3}, y_{1}\right) \Gamma_{\uparrow \uparrow}\left(y_{2}, y_{4} ; y_{6}, x^{\prime}\right) \\
& \left.\quad+\frac{1}{2} \Gamma_{\uparrow \uparrow}\left(x^{\prime}, y_{5} ; y_{3}, y_{1}\right) \Gamma_{\uparrow \uparrow}\left(y_{2}, y_{4} ; y_{6}, x\right)+\frac{1}{2} \Gamma_{\uparrow \uparrow}\left(x, y_{6} ; y_{4}, y_{2}\right) \Gamma_{\uparrow \uparrow}\left(y_{1}, y_{3} ; y_{5}, x^{\prime}\right)\right] .
\end{aligned}
$$

Here we note that the full vertex function $\Gamma_{\sigma_{4} \sigma_{3} ; \sigma_{2} \sigma_{1}}\left(x_{4}, x_{3} ; x_{2}, x_{1}: i \varepsilon_{4}, i \varepsilon_{3} ; i \varepsilon_{2}, i \varepsilon_{1}\right)$ is antisymmetric with respect to interchanges of the arguments (together with the spin suffixes) in the first or second pair: 1 and 2, or 3 and 4.29 In the symmetrized expression, Eq. (29), the eight terms in the bracket consist of four pairs each of which is connected by the symmetry operation for the time reversal; $\left(x_{4}, x_{3} ; x_{2}, x_{1}\right) \Leftrightarrow\left(x_{1}, x_{2} ; x_{3}, x_{4}\right)$. Consequently, the imaginary part of the proper self-energy is proportional to $\varepsilon^{2}$ and Eq. (13) holds at $T=0$ as long as the integral in Eq. (29) is finite, i.e., in the case the perturbation theory is valid. 


\section{CONDUCTANCE}

In this section, we consider the expression of the dc conductance $g$ applying the perturbation analysis 0 to the current-current correlation function. We show that the contributions of the vertex correction to $g$ is zero at $T=0$ when the currents are measured in the noninteracting leads. The resultant expression, Eq. (44), is written in terms of the transmission coefficient for the single-particle-like excitation at the Fermi energy, $t(0)$, defined by Eq. (11).

When the electric field is applied to the central region, the dc conductance within the Kubo formalism is given by

$$
g=\lim _{\omega \rightarrow 0} \frac{K\left(x, x^{\prime} ; \omega+i 0^{+}\right)-K\left(x, x^{\prime} ; i 0^{+}\right)}{i \omega},
$$

where

$$
\begin{gathered}
K\left(x, x^{\prime} ; \omega+i 0^{+}\right)=i \int_{0}^{\infty} d t\left\langle\left[j(x, t), j\left(x^{\prime}, 0\right)\right]\right\rangle e^{i\left(\omega+i 0^{+}\right) t}, \\
j(x)=\sum_{\sigma} \frac{e}{2 m i}\left[\psi_{\sigma}^{\dagger}(x) \frac{\partial \psi_{\sigma}(x)}{\partial x}-\frac{\partial \psi_{\sigma}^{\dagger}(x)}{\partial x} \psi_{\sigma}(x)\right] .
\end{gathered}
$$

As discussed by Fisher \& Lee, 10,11 $x$ and $x^{\prime}$ are arbitrary in Eq. (30) and $g$ is independent of the choice of the positions $x$ and $x^{\prime}$ owing to the current conservation specific to one dimension. This feature can be seen explicitly in the Lehmann representation

$$
g=\frac{\pi \beta}{Z} \sum_{\alpha \alpha^{\prime}} e^{-\beta E_{\alpha}}\left\langle\alpha|j(x)| \alpha^{\prime}\right\rangle\left\langle\alpha^{\prime}\left|j\left(x^{\prime}\right)\right| \alpha\right\rangle \delta\left(E_{\alpha}-E_{\alpha^{\prime}}\right),
$$

where $Z \equiv \operatorname{Tr} e^{-\beta \mathcal{H}},|\alpha\rangle$ is the eigenstate of $\mathcal{H}$, and $E_{\alpha}$ is its eigenvalue. For obtaining this representation, we have used the relation caused by the time reversal symmetry: $K\left(x, x^{\prime} ; z\right)=K\left(x^{\prime}, x ; z\right)$ and

$$
\left[K\left(x, x^{\prime} ; \omega+i 0^{+}\right)-K\left(x, x^{\prime} ; \omega-i 0^{+}\right)\right] / 2 i=\operatorname{Im} K\left(x, x^{\prime} ; \omega+i 0^{+}\right) .
$$

In Eq. (33), the matrix element $\left\langle\alpha|j(x)| \alpha^{\prime}\right\rangle$ is independent of $x$ because $E_{\alpha}=E_{\alpha^{\prime}} .10$ This can be confirmed by multiplying the equation of continuity $\partial \rho(x, t) / \partial t+\partial j(x, t) / \partial x=0$ by $\langle\alpha|$ on the left and by $\left|\alpha^{\prime}\right\rangle$ on the right, where $\rho \equiv e \sum_{\sigma} \psi_{\sigma}^{\dagger} \psi_{\sigma}$. 
In the Kubo formalism, Eq. (30), the dc conductance is obtained from the $\omega$-linear imaginary part of $K\left(x, x^{\prime} ; \omega+i 0^{+}\right)$. In addition, Eq. (34) means that the imaginary part corresponds to the discontinuity of $K\left(x, x^{\prime} ; z\right)$ at the real axis in the complex $z$-plane, i.e., the singular contribution of the form $\operatorname{sgn}(\operatorname{Im} z)$. This type of singular contribution in the correlation function can be extracted using the perturbation analysis which was originally used by Shiba to derive the Korringa relation in Kondo alloys. 5 In the previous study, we applied this method to the conductance through a single Anderson-Wolff impurity 220 In order to carry out the analysis, we introduce the Matsubara function

$$
K\left(x, x^{\prime} ; i \nu_{n}\right)=\int_{0}^{\beta} d \tau\left\langle T_{\tau} j^{M}(x, \tau) j^{M}\left(x^{\prime}, 0\right)\right\rangle e^{i \nu_{n} \tau},
$$

where $\nu_{n}=2 \pi n / \beta$. The $\omega$-linear imaginary part of $K\left(x, x^{\prime} ; \omega+i 0^{+}\right)$is obtained from the $|\nu|$-linear contribution of $K\left(x, x^{\prime} ; i \nu\right)$ because $|\nu|=\nu \operatorname{sgn} \nu$ is replaced by $-i \omega$ by the analytic continuation $i \nu \rightarrow \omega+i 0^{+}$. The Matsubara function $K\left(x, x^{\prime} ; i \nu\right)$ can be represented by a sum of the diagrams shown in Fig. 1 , and the corresponding expression for $T=0$ is written, replacing sums over discrete Matsubara frequencies by integrals, as

$$
\begin{aligned}
K\left(x, x^{\prime} ; i \nu\right)= & K^{(a)}\left(x, x^{\prime} ; i \nu\right)+K^{(b)}\left(x, x^{\prime} ; i \nu\right), \\
K^{(a)}\left(x, x^{\prime} ; i \nu\right)= & -\left(\frac{e}{2 m i}\right)^{2}\left\{\partial / \partial x_{4}-\partial / \partial x_{1}\right\}\left\{\partial / \partial x_{3}-\partial / \partial x_{2}\right\} \\
& \times\left.\sum_{\sigma} \int_{-\infty}^{+\infty} \frac{d \varepsilon}{2 \pi} G\left(x_{3}, x_{1} ; i \varepsilon\right) G\left(x_{4}, x_{2} ; i \varepsilon+i \nu\right)\right|_{\substack{x_{1}, x_{2} \rightarrow x \\
x_{2}, x_{3} \rightarrow x^{\prime}}}, \\
K^{(b)}\left(x, x^{\prime} ; i \nu\right)= & -\left(\frac{e}{2 m i}\right)^{2}\left\{\partial / \partial x_{4}-\partial / \partial x_{1}\right\}\left\{\partial / \partial x_{3}-\partial / \partial x_{2}\right\} \\
& \times \sum_{\sigma \sigma^{\prime}} \int_{-\infty}^{+\infty} \frac{d \varepsilon d \varepsilon^{\prime}}{(2 \pi)^{2}} \int_{0}^{L} \cdots \int_{0}^{L} \prod_{i=1}^{4} d y_{i} G\left(y_{1}, x_{1} ; i \varepsilon\right) G\left(x_{4}, y_{4} ; i \varepsilon+i \nu\right) \\
& \times \Gamma_{\sigma \sigma^{\prime} ; \sigma^{\prime} \sigma}\left(y_{4}, y_{3} ; y_{2}, y_{1}: i \varepsilon+i \nu, i \varepsilon^{\prime} ; i \varepsilon^{\prime}+i \nu, i \varepsilon\right) \\
& \times\left. G\left(x_{3}, y_{3} ; i \varepsilon^{\prime}\right) G\left(y_{2}, x_{2} ; i \varepsilon^{\prime}+i \nu\right)\right|_{\substack{x_{1}, x_{4} \rightarrow x \\
x_{2}, x_{3} \rightarrow x^{\prime}}} .
\end{aligned}
$$

In what follows we choose $x$ to be in the right lead and $x^{\prime}$ to be in the left lead, and thus $x^{\prime}<0<y_{i}<L<x$ with $i=1, \ldots, 4$. Then the asymptotic form of the Matsubara Green's functions in Eqs. (37) and (38) can be written, making use of Eqs. (4) and (19)-(21), as $G\left(x, y_{i} ; i \varepsilon\right) \propto \exp \left[i \kappa(i \varepsilon)\left(x-y_{i}\right)\right]$ and $G\left(x^{\prime}, y_{i} ; i \varepsilon\right) \propto \exp \left[i \kappa(i \varepsilon)\left(y_{i}-x^{\prime}\right)\right]$. Therefore the 
derivative with respect to $x_{1}, \ldots, x_{4}$ can be done explicitly, and the expression valid for $x^{\prime}<0$ and $L<x$ is obtained as

$$
\begin{aligned}
K^{(a)}\left(x, x^{\prime} ; i \nu\right)= & -\left(\frac{e}{2 m}\right)^{2} \sum_{\sigma} \int_{-\infty}^{+\infty} \frac{d \varepsilon}{2 \pi}\{\kappa(i \varepsilon+i \nu)-\kappa(i \varepsilon)\}^{2} G\left(x^{\prime}, x ; i \varepsilon\right) G\left(x, x^{\prime} ; i \varepsilon+i \nu\right) \\
K^{(b)}\left(x, x^{\prime} ; i \nu\right)= & -\left(\frac{e}{2 m}\right)^{2} \sum_{\sigma \sigma^{\prime}} \int_{-\infty}^{+\infty} \frac{d \varepsilon d \varepsilon^{\prime}}{(2 \pi)^{2}} \int_{0}^{L} \cdots \int_{0}^{L} \prod_{i=1}^{4} d y_{i} \\
& \times\{\kappa(i \varepsilon+i \nu)-\kappa(i \varepsilon)\} G\left(y_{1}, x ; i \varepsilon\right) G\left(x, y_{4} ; i \varepsilon+i \nu\right) \\
& \times \Gamma_{\sigma \sigma^{\prime} ; \sigma^{\prime} \sigma}\left(y_{4}, y_{3} ; y_{2}, y_{1}: i \varepsilon+i \nu, i \varepsilon^{\prime} ; i \varepsilon^{\prime}+i \nu, i \varepsilon\right) \\
& \times\left\{\kappa\left(i \varepsilon^{\prime}+i \nu\right)-\kappa\left(i \varepsilon^{\prime}\right)\right\} G\left(x^{\prime}, y_{2} ; i \varepsilon^{\prime}\right) G\left(y_{3}, x^{\prime} ; i \varepsilon^{\prime}+i \nu\right)
\end{aligned}
$$

We can now extract the singular $\nu \operatorname{sgn} \nu$ contribution from Eqs. (39) and (40). Shiba has shown precisely that this type of singular contribution comes only from the diagrams with two intermediate Green's functions which carry the same frequencies in the limit $\nu \rightarrow$ 0. 0.5 The simplest example is the diagram Fig. 4 (a) in which the frequencies are assigned as $G(i \varepsilon) G(i \varepsilon+i \nu)$. The $\nu \operatorname{sgn} \nu$ contribution is originated from the $\operatorname{sgn} \varepsilon$ term in the Matsubara Green's function: for instance, see Eq. (20) for the free Green's function. The full Green's function can be expressed, by separating the singular and regular parts, as

$$
G\left(x, x^{\prime} ; i \varepsilon\right)=G^{\prime}\left(x, x^{\prime} ; i \varepsilon\right)+i G^{\prime \prime}\left(x, x^{\prime} ; i \varepsilon\right) \operatorname{sgn} \varepsilon
$$

Note that, when $\varepsilon=0, G^{\prime}\left(x, x^{\prime} ; 0\right)$ and $G^{\prime \prime}\left(x, x^{\prime} ; 0\right)$ correspond to the real and imaginary parts of the retarded Green's function $G\left(x, x^{\prime} ; i 0^{+}\right)$owing to the symmetry property Eq. (6). The $\nu \operatorname{sgn} \nu$ contribution of $K\left(x, x^{\prime} ; i \nu\right)$ due to the two intermediate Green's functions appears through the integration of the product $\operatorname{sgn}(\varepsilon+\nu) \operatorname{sgn}(\varepsilon)$ over $\varepsilon$;

$$
\left.\int_{-\infty}^{+\infty} d \varepsilon A(i \varepsilon) \operatorname{sgn}(\varepsilon+\nu) \operatorname{sgn}(\varepsilon)\right|_{\nu \rightarrow 0}=\int_{-\infty}^{+\infty} d \varepsilon A(i \varepsilon)-2 A(0) \nu \operatorname{sgn} \nu .
$$

Here $A(i \varepsilon)$ is a smooth function which can be expressed in terms of $G^{\prime}, G^{\prime \prime}$, and so on, and the second term in the right-hand side appears as a result of the derivative $d \operatorname{sgn}(\varepsilon+\nu) / d \nu=$ $2 \delta(\varepsilon+\nu)$. Thus, the next thing we should do is to calculate the coefficient $A(0)$ taking into account the vertex correction. In the perturbation theory, the diagrams which contain 
the two intermediate Green's functions can be classified into four groups[see Fig. 5. 5 . In the figure, the two intermediate Green's functions which yield the singular contribution are marked with the cross, and the external frequency $\nu$ is taken to be zero in the remaining part of each diagrams. One remarkable feature is that the contributions of the diagrams (b1)-(b3) become zero, i.e., the coefficient $A(0)$ corresponding to these diagrams is zero. This is because the each of diagrams (b1)-(b3) contains at least one current vertex $\left\{\kappa\left(i \varepsilon^{\prime}+i \nu\right)-\kappa\left(i \varepsilon^{\prime}\right)\right\}$, for which $\nu$ can be simply taken to be zero. In contrast to (b1)-(b3), the contribution of the diagram (a) is finite because the two intermediate Green's functions and the current vertex carry the same the internal frequency $\varepsilon$, and the product $\operatorname{sgn}(\varepsilon+\nu) \operatorname{sgn}(\varepsilon)$ appears also from the square of the current vertex [see Eq. (39)]. Therefore, the finite contribution of the $\nu \operatorname{sgn} \nu$ term comes only from the diagram (a), which can be calculated by substituting Eqs. (20), (21) and (41) into Eq. (39). Consequently, the dc conductance at $T=0$ is obtained, by reinserting $\hbar$, as

$$
g=\frac{e^{2}}{\pi \hbar} \frac{1}{[\pi \rho(0)]^{2}}\left|G\left(x, x^{\prime} ; i 0^{+}\right)\right|^{2},
$$

where $x$ and $x^{\prime}$ is taken to be $x^{\prime}<0$ and $L<x$ as already noted. This expression can be rewritten in terms of the transmission coefficient $t(0)$ defined by Eqs. (8) and (11);

$$
g=\frac{2 e^{2}}{h}|t(0)|^{2}
$$

These results Eqs. (43) and (44) are generalized to a quasi-one-dimensional system with a number of channels in the next section. Furthermore, the present analysis can be applied to a tight-binding model and the results can also be generalized to the system on the lattice 30

Before closing this section we consider briefly the reason why the contributions of the vertex correction disappear, and it seems to be understood in relation to the back flow in the leads. In the usual Fermi liquid theory the back flow can be represented in terms of the three point vertex function for the current, 27,28 which is one parts of Eq. (38) and can be written as [see Fig. 6]

$\Lambda\left(x^{\prime} ; y_{4}, y_{1} ; i \varepsilon+i \nu, i \varepsilon\right)=\sum_{\sigma^{\prime}} \int_{-\infty}^{+\infty} \frac{d \varepsilon^{\prime}}{2 \pi} \int_{0}^{L} \int_{0}^{L} d y_{2} d y_{3} \Gamma_{\sigma \sigma^{\prime} ; \sigma^{\prime} \sigma}\left(y_{4}, y_{3} ; y_{2}, y_{1}: i \varepsilon+i \nu, i \varepsilon^{\prime} ; i \varepsilon^{\prime}+i \nu, i \varepsilon\right)$ 


$$
\times\left.\frac{e}{2 m i}\left\{\partial / \partial x_{3}-\partial / \partial x_{2}\right\} G\left(x_{3}, y_{3} ; i \varepsilon^{\prime}\right) G\left(y_{2}, x_{2} ; i \varepsilon^{\prime}+i \nu\right)\right|_{x_{2}, x_{3} \rightarrow x^{\prime}} .
$$

Specifically, the value $\Lambda\left(x^{\prime} ; y_{4}, y_{1} ; 0,0\right)$ for zero frequencies can be related to the renormalization of the current caused by the back flow.27,28 Since we are considering the system without the translational invariance, a more precise definition of the back flow should be necessary for a general argument. Nevertheless, we can show the value $\Lambda\left(x^{\prime} ; y_{4}, y_{1} ; 0,0\right)$ to be zero when $x^{\prime}$ belongs to the noninteracting leads. It can be shown by performing the derivative with respect to $x_{2}, x_{3}$ as it was done for obtaining Eq. (40);

$$
\begin{aligned}
\Lambda\left(x^{\prime} ; y_{4}, y_{1} ; i \varepsilon+i \nu, i \varepsilon\right)= & \pm \sum_{\sigma^{\prime}} \int_{-\infty}^{+\infty} \frac{d \varepsilon^{\prime}}{2 \pi} \int_{0}^{L} \int_{0}^{L} d y_{2} d y_{3} \Gamma_{\sigma \sigma^{\prime} ; \sigma^{\prime} \sigma}\left(y_{4}, y_{3} ; y_{2}, y_{1}: i \varepsilon+i \nu, i \varepsilon^{\prime} ; i \varepsilon^{\prime}+i \nu, i \varepsilon\right) \\
& \times \frac{e}{2 m}\left\{\kappa\left(i \varepsilon^{\prime}+i \nu\right)-\kappa\left(i \varepsilon^{\prime}\right)\right\} G\left(y_{2}, x^{\prime} ; i \varepsilon^{\prime}+i \nu\right) G\left(x^{\prime}, y_{3} ; i \varepsilon^{\prime}\right),
\end{aligned}
$$

where \pm is chosen to be + for $x^{\prime}<0$, and - for $L<x^{\prime}$. Since the current vertex $\left\{\kappa\left(i \varepsilon^{\prime}+i \nu\right)-\kappa\left(i \varepsilon^{\prime}\right)\right\}$ is zero for $\nu=0$, the three point function becomes $\Lambda\left(x^{\prime} ; y_{4}, y_{1} ; 0,0\right)=0$.

In this sense, the current is not renormalized and the effect due to the back flow is absent in the noninteracting leads. This is caused by the fact that the asymptotic form of the Green's function in the leads is given by a simple superposition of the incident, transmitted, and reflected waves [see Eqs. (8) and (9)].

\section{EXTENSION TO HIGHER DIMENSIONS}

So far, we have considered the system in one dimension. In this section, we generalize the results obtained in the above to the quasi-one-dimensional system, which is finite in the direction perpendicular to the $x$-direction [the transverse direction $\boldsymbol{\rho}$ ]. The extension can be done following along almost the same lines as those for the one-dimensional system. This is because the correspondence of the the imaginary part and the discontinuity of the Green's function holds also for the quasi-one-dimensional system, Eq. (52), owing to the time reversal symmetry. So, the analysis of the Green's function used in Sec. IIV is also available here, and thus we will present only the outline of the extension. In Sec. VA, we 
introduce the transmission and reflection coefficients for a number of scattering channels, Eq. (55), making use of the the single-particle Green's function, and examine the unitarity condition for the coefficients generalizing the discussion in Sec. II. In Sec. VB, we consider the contributions of the vertex correction to the dc conductance in the quasi-one-dimensional system generalizing the calculations performed in Sec. $\mathbb{}$, and as a result the conductance is expressed in terms of the single-particle Green's function or the transmission coefficient; Eqs. (69) or (70).

\section{A. Scattering Coefficients}

In this subsection, we generalize the definition of the transmission and reflection coefficients discussed in Sec. [1] to the quasi-one-dimensional system. We now introduce a set of normal modes $\chi_{a}(\boldsymbol{\rho})$ satisfying the Schrödinger equation for the transverse direction in the leads

$$
\left[-\frac{1}{2 m} \frac{\partial^{2}}{\partial \boldsymbol{\rho}^{2}}+V_{c}(\boldsymbol{\rho})\right] \chi_{a}(\boldsymbol{\rho})=\epsilon_{a} \chi_{a}(\boldsymbol{\rho}),
$$

where $a$ is the subband index, $\epsilon_{a}$ is the subband energy, and $V_{c}(\boldsymbol{\rho})$ is confinement potential for the transverse direction which is perpendicular to the $x$-direction. In what follows we choose the normal modes to be real, i.e., $\chi_{a}(\boldsymbol{\rho})=\chi_{a}^{*}(\boldsymbol{\rho})$. This is possible because of the time reversal symmetry of Eq. (47), and is convenient for the discussion in the following. The free Green's function is diagonal in the subband indices

$$
G_{0}\left(\boldsymbol{r}, \boldsymbol{r}^{\prime} ; z\right)=\sum_{a} \chi_{a}(\boldsymbol{\rho}) \chi_{a}\left(\boldsymbol{\rho}^{\prime}\right) G_{a}^{(0)}\left(x, x^{\prime} ; z\right)
$$

where $\boldsymbol{r}=(x, \boldsymbol{\rho}), \boldsymbol{r}^{\prime}=\left(x^{\prime}, \boldsymbol{\rho}^{\prime}\right)$, and $G_{a}^{(0)}\left(x, x^{\prime} ; z\right)$ is the free Green's function for the one dimension, which is obtained by replacing the chemical potential $\mu$ by $\mu-\epsilon_{a}$ in the right-hand side of Eqs. (7) or (19)-(21). When the Coulomb interaction and the scattering potential are restricted for the electrons staying in the central region $0 \leq x \leq L$, the Dyson equation is written, by generalizing Eq. (41), as 


$$
\begin{aligned}
G_{b a}\left(x, x^{\prime} ; z\right)= & G_{b}^{(0)}\left(x, x^{\prime} ; z\right) \delta_{b a} \\
& +\int_{0}^{L} \int_{0}^{L} d x_{1} d x_{2} G_{b}^{(0)}\left(x, x_{1} ; z\right) \mathcal{T}_{b a}\left(x_{1}, x_{2} ; z\right) G_{a}^{(0)}\left(x_{2}, x^{\prime} ; z\right) .
\end{aligned}
$$

Here the subscript ba denotes the subband indices defined by

$$
G_{b a}\left(x, x^{\prime} ; z\right) \equiv \iint d \boldsymbol{\rho} d \boldsymbol{\rho}^{\prime} \chi_{b}(\boldsymbol{\rho}) G\left(\boldsymbol{r}, \boldsymbol{r}^{\prime} ; z\right) \chi_{a}\left(\boldsymbol{\rho}^{\prime}\right)
$$

Because of the time-reversal symmetry, the relation corresponding to Eq. (5) holds also for the quasi-one-dimensional system as $G\left(\boldsymbol{r}^{\prime}, \boldsymbol{r} ; z\right)=G\left(\boldsymbol{r}, \boldsymbol{r}^{\prime} ; z\right)$. Thus using Eq. (50), we obtain

$$
G_{a b}\left(x, x^{\prime} ; z\right)=G_{b a}\left(x^{\prime}, x ; z\right)
$$

Therefore, as in the one-dimensional case, the discontinuity of the Green's function at the real axis in the complex $z$-plane corresponds to the imaginary part;

$$
\left[G_{a b}\left(x, x^{\prime} ; \varepsilon+i 0^{+}\right)-G_{a b}\left(x, x^{\prime} ; \varepsilon-i 0^{+}\right)\right] / 2 i=\operatorname{Im} G_{a b}\left(x, x^{\prime} ; \varepsilon+i 0^{+}\right)
$$

Owing to this property, we can obtain the imaginary part using the perturbation analysis for extracting the singular contributions.

When both $x$ and $x^{\prime}$ belong the leads, the asymptotic form of the full Green's function can be written explicitly making use of Eq. (49). So, the equation corresponding to Eq. (8) is written by taking the values of $x^{\prime}$ and $x$ to be $x^{\prime}<0$ and $L<x$;

$$
G_{b a}\left(x, x^{\prime} ; i 0^{+}\right)=e^{i k_{b} x}\left[\delta_{b a}-\frac{i}{v_{b}} \mathcal{T}_{b a: k_{b} k_{a}}\left(i 0^{+}\right)\right]\left(-\frac{i}{v_{a}}\right) e^{-i k_{a} x^{\prime}},
$$

where the frequency is chosen to be $z=i 0^{+}, k_{a} \equiv \sqrt{2 m\left(\mu-\epsilon_{a}\right)}$ is the wave number of single-particle-like excitation at Fermi energy, and $v_{a} \equiv k_{a} / m$ is the velocity. In Eq. (53) the initial and final states, $a$ and $b$, are assumed to be propagating modes with real values of the wave number. $\mathcal{T}_{b a: k_{b} k_{a}}(z)$ is the Fourier transform of $\mathcal{T}_{b a}\left(x_{1}, x_{2} ; z\right)$ with respect to $x_{1}$ and $x_{2}$ [see Eq. (10)]. Similarly, the equation corresponding to Eq. (9) is written by taking the positions to be $x^{\prime}<x<0$;

$$
G_{b a}\left(x, x^{\prime} ; i 0^{+}\right)=\left[\delta_{b a} e^{i k_{a} x}-\frac{i}{v_{b}} \mathcal{T}_{b a:-k_{b} k_{a}}\left(i 0^{+}\right) e^{-i k_{b} x}\right]\left(-\frac{i}{v_{a}}\right) e^{-i k_{a} x^{\prime}}
$$


Since the retarded Green's function $G_{b a}\left(x, x^{\prime} ; i 0^{+}\right)$represents a propagation of a singleparticle-like excitation at the Fermi energy as discussed in Sec. II], it seems natural to define the scattering coefficients making use of Eqs. (53) and (54) as

$$
\begin{aligned}
t_{b a} & \equiv\left[\delta_{b a}-\frac{i}{v_{b}} \mathcal{T}_{b a: k_{b} k_{a}}\left(i 0^{+}\right)\right] \sqrt{\frac{v_{b}}{v_{a}}} \\
r_{b a} & \equiv-\frac{i}{v_{b}} \mathcal{T}_{b a:-k_{b} k_{a}}\left(i 0^{+}\right) \sqrt{\frac{v_{b}}{v_{a}}}
\end{aligned}
$$

where a factor $\sqrt{v_{b} / v_{a}}$ is introduced for the normalization. Then the unitarity condition, $\sum_{b}^{\prime}\left[\left|t_{b a}\right|^{2}+\left|r_{b a}\right|^{2}\right]=1$, is expressed in the form

$$
\operatorname{Im} \mathcal{T}_{a a: k_{a} k_{a}}\left(i 0^{+}\right)=-\sum_{b}^{\prime} \frac{1}{2 v_{b}}\left[\left|\mathcal{T}_{b a: k_{b} k_{a}}\left(i 0^{+}\right)\right|^{2}+\left|\mathcal{T}_{b a:-k_{b} k_{a}}\left(i 0^{+}\right)\right|^{2}\right],
$$

where the primed sum runs over the propagating modes. At $T=0$, the unitarity condition holds for the excitation at the Fermi energy if the imaginary part of the proper self-energy is zero;

$$
\operatorname{Im} \Sigma_{a b}\left(x, x^{\prime} ; i 0^{+}\right)=0
$$

As in the one-dimensional case, Eq. (57) can be derived by using the operator identity Eq. (15). In the present case the matrix element of the operator $\mathcal{T}$ is defined by $\left\langle b, k_{b}|\mathcal{T}| a, k_{a}\right\rangle \equiv$ $\mathcal{T}_{b a: k_{b} k_{a}}\left(i 0^{+}\right)$, and the product of the operators is understood to be an integral for the motion in the $x$ direction and a sum over the subband index. The operator for the proper self-energy $\Sigma$ is Hermitian owing to Eq. (58). Thus Eq. (57) can be obtained from the diagonal element of the identity in the wave-number indices, which is written by generalizing Eq. (16) as $\mathcal{T}_{a a: k_{a} k_{a}}\left(i 0^{+}\right)-\mathcal{T}_{a a: k_{a} k_{a}}^{*}\left(i 0^{+}\right)=-\sum_{b} \int_{-\infty}^{+\infty} \frac{d k^{\prime}}{2 \pi} \mathcal{T}_{b a: k^{\prime} k_{a}}^{*}\left(i 0^{+}\right) 2 \pi i \delta\left(k^{\prime 2} / 2 m+\epsilon_{b}-\mu\right) \mathcal{T}_{b a: k^{\prime} k_{a}}\left(i 0^{+}\right)$.

Because of the energy conservation due to the delta function, the sum over $b$ is restricted to the propagating modes and thus we obtain Eq. (57). We note that Eq. (58) can also be derived in the perturbation theory by extending the calculation of the imaginary part performed in the Sec. III; by including sums over subband indices for intermediate states and using Eq. (52). 


\section{B. Conductance}

Next we consider the expression of the conductance by extending the calculation performed in Sec. IV. In the present case, the operator for the current in the $x$ direction is given by

$$
J(x)=\sum_{\sigma} \int d \boldsymbol{\rho} \frac{e}{2 m i}\left[\psi_{\sigma}^{\dagger}(x, \boldsymbol{\rho}) \frac{\partial \psi_{\sigma}(x, \boldsymbol{\rho})}{\partial x}-\frac{\partial \psi_{\sigma}^{\dagger}(x, \boldsymbol{\rho})}{\partial x} \psi_{\sigma}(x, \boldsymbol{\rho})\right] .
$$

When the electric field is applied to the central region along the $x$ direction, the dc conductance within the Kubo formalism is given by

$$
\begin{aligned}
& g=\lim _{\omega \rightarrow 0} \frac{\widetilde{K}\left(x, x^{\prime} ; \omega+i 0^{+}\right)-\widetilde{K}\left(x, x^{\prime} ; i 0^{+}\right)}{i \omega}, \\
& \widetilde{K}\left(x, x^{\prime} ; \omega+i 0^{+}\right) \equiv i \int_{0}^{\infty} d t\left\langle\left[J(x, t), J\left(x^{\prime}, 0\right)\right]\right\rangle e^{i\left(\omega+i 0^{+}\right) t} .
\end{aligned}
$$

where $x$ and $x^{\prime}$ are arbitrary owing to the current conservation. In the Kubo formalism the dc conductance is obtained from the $\omega$-linear imaginary part of $\widetilde{K}\left(x, x^{\prime} ; \omega+i 0^{+}\right)$. In addition, the $\omega$-linear imaginary part corresponds to the $\nu \operatorname{sgn} \nu$ term in the Matsubara function $\widetilde{K}\left(x, x^{\prime} ; i \nu\right)$ because the relation Eq. (34) also holds for $\widetilde{K}$ owing to the symmetry

property $\widetilde{K}\left(x, x^{\prime} ; z\right)=\widetilde{K}\left(x^{\prime}, x ; z\right)$. Therefore, the conductance is obtained by extracting the $\nu \operatorname{sgn} \nu$ term using the diagrammatic analysis. At $T=0$, the equations corresponding to Eqs. (36)-(38) are written as

$$
\begin{aligned}
\widetilde{K}\left(x, x^{\prime} ; i \nu\right)= & \widetilde{K}^{(a)}\left(x, x^{\prime} ; i \nu\right)+\widetilde{K}^{(b)}\left(x, x^{\prime} ; i \nu\right) \\
\widetilde{K}^{(a)}\left(x, x^{\prime} ; i \nu\right)= & -\left(\frac{e}{2 m i}\right)^{2}\left\{\partial / \partial x_{4}-\partial / \partial x_{1}\right\}\left\{\partial / \partial x_{3}-\partial / \partial x_{2}\right\} \\
& \times\left.\sum_{\sigma} \sum_{a b} \int_{-\infty}^{+\infty} \frac{d \varepsilon}{2 \pi} G_{a b}\left(x_{3}, x_{1} ; i \varepsilon\right) G_{b a}\left(x_{4}, x_{2} ; i \varepsilon+i \nu\right)\right|_{\substack{x_{1}, x_{4} \rightarrow x \\
x_{2}, x_{3} \rightarrow x^{\prime}}}, \\
\widetilde{K}^{(b)}\left(x, x^{\prime} ; i \nu\right)= & -\left(\frac{e}{2 m i}\right)^{2}\left\{\partial / \partial x_{4}-\partial / \partial x_{1}\right\}\left\{\partial / \partial x_{3}-\partial / \partial x_{2}\right\} \\
& \times \sum_{\sigma \sigma^{\prime}} \sum_{a b} \sum_{a_{1} a_{2} a_{4}} \int_{-\infty}^{+\infty} \frac{d \varepsilon d \varepsilon^{\prime}}{(2 \pi)^{2}} \int_{0}^{L} \ldots \int_{0}^{L} \prod_{i=1}^{4} d y_{i} G_{a_{1} b}\left(y_{1}, x_{1} ; i \varepsilon\right) G_{b a_{4}}\left(x_{4}, y_{4} ; i \varepsilon+i \nu\right) \\
& \times \Gamma_{\sigma \sigma^{\prime} ; \sigma^{\prime} \sigma}^{a_{4} a_{3} ; a_{1}}\left(y_{4}, y_{3} ; y_{2}, y_{1}: i \varepsilon+i \nu, i \varepsilon^{\prime} ; i \varepsilon^{\prime}+i \nu, i \varepsilon\right) \\
& \times\left. G_{a a_{3}}\left(x_{3}, y_{3} ; i \varepsilon^{\prime}\right) G_{a_{2} a}\left(y_{2}, x_{2} ; i \varepsilon^{\prime}+i \nu\right)\right|_{\substack{x_{1}, x_{4} \rightarrow x \\
x_{2}, x_{3} \rightarrow x^{\prime}}}
\end{aligned}
$$


Here superscripts of the vertex function denote the subband indices defined by

$$
\Gamma_{\sigma \sigma^{\prime} ; \sigma^{\prime} \sigma}^{a_{4} a_{3} ; a_{2} a_{1}}\left(x_{4}, x_{3} ; x_{2}, x_{1}\right) \equiv \int \prod_{i=1}^{4} d \boldsymbol{\rho}_{\boldsymbol{i}} \chi_{a_{4}}\left(\boldsymbol{\rho}_{\mathbf{4}}\right) \chi_{a_{3}}\left(\boldsymbol{\rho}_{\mathbf{3}}\right) \Gamma_{\sigma \sigma^{\prime} ; \sigma^{\prime} \sigma}\left(\boldsymbol{r}_{\mathbf{4}}, \boldsymbol{r}_{\mathbf{3}} ; \boldsymbol{r}_{\mathbf{2}}, \boldsymbol{r}_{\mathbf{1}}\right) \chi_{a_{2}}\left(\boldsymbol{\rho}_{\mathbf{2}}\right) \chi_{a_{1}}\left(\boldsymbol{\rho}_{\mathbf{1}}\right)
$$

In what follows we choose $x$ to be in the right lead and $x^{\prime}$ to be in the left lead, and thus $x^{\prime}<0<y_{i}<L<x$ with $i=1, \ldots, 4$. Then, the derivative with respect to $x_{1} \ldots, x_{4}$ can be performed explicitly making use of the asymptotic form of the Green's function, and the equations corresponding to Eqs. (39) and (40) can be obtained as

$$
\begin{aligned}
\widetilde{K}^{(a)}\left(x, x^{\prime} ; i \nu\right)= & -\left(\frac{e}{2 m}\right)^{2} \sum_{\sigma} \sum_{a b} \int_{-\infty}^{+\infty} \frac{d \varepsilon}{2 \pi}\left\{\kappa_{b}(i \varepsilon+i \nu)-\kappa_{b}(i \varepsilon)\right\}\left\{\kappa_{a}(i \varepsilon+i \nu)-\kappa_{a}(i \varepsilon)\right\} \\
& \times G_{a b}\left(x^{\prime}, x ; i \varepsilon\right) G_{b a}\left(x, x^{\prime} ; i \varepsilon+i \nu\right) \\
\widetilde{K}^{(b)}\left(x, x^{\prime} ; i \nu\right)= & -\left(\frac{e}{2 m}\right)^{2} \sum_{\sigma \sigma^{\prime}} \sum_{a b} \sum_{a_{1} a_{2}} \int_{-\infty}^{+\infty} \frac{d \varepsilon d \varepsilon^{\prime}}{(2 \pi)^{2}} \int_{0}^{L} \cdots \int_{0}^{L} \prod_{i=1}^{4} d y_{i} \\
& \times\left\{\kappa_{b}(i \varepsilon+i \nu)-\kappa_{b}(i \varepsilon)\right\} G_{a_{1} b}\left(y_{1}, x ; i \varepsilon\right) G_{b a_{4}}\left(x, y_{4} ; i \varepsilon+i \nu\right) \\
& \times \Gamma_{\sigma \sigma^{\prime} ; \sigma^{\prime} \sigma}^{a_{4} a_{3} a_{2} a_{1}}\left(y_{4}, y_{3} ; y_{2}, y_{1}: i \varepsilon+i \nu, i \varepsilon^{\prime} ; i \varepsilon^{\prime}+i \nu, i \varepsilon\right) \\
& \times\left\{\kappa_{a}\left(i \varepsilon^{\prime}+i \nu\right)-\kappa_{a}\left(i \varepsilon^{\prime}\right)\right\} G_{a a_{3}}\left(x^{\prime}, y_{3} ; i \varepsilon^{\prime}\right) G_{a_{2} a}\left(y_{2}, x^{\prime} ; i \varepsilon^{\prime}+i \nu\right) .
\end{aligned}
$$

Here $\kappa_{a}(i \varepsilon)$ denotes the quantity which is given by replacing $\mu$ by $\mu-\epsilon_{a}$ in Eqs. (20) and (21). At $T=0$, the contributions of the vertex correction, Eq. (68), to the dc conductance becomes zero as that in the one-dimensional case. This is because the current vertex $\left\{\kappa_{a}\left(i \varepsilon^{\prime}+\right.\right.$ $\left.i \nu)-\kappa_{a}\left(i \varepsilon^{\prime}\right)\right\}$ is zero for $\nu=0$. This feature can also be understood in terms of the three point vertex function for $J\left(x^{\prime}\right)$, which represents the renormalization of the current or the effects of the back flow as discussed in the last part of Sec. $\mathbb{\nabla}$ [see Fig. 6]. Consequently, the dc conductance is determined by the $\nu \operatorname{sgn} \nu$ term in the right-hand side of Eq. (67), and the expression of the dc conductance for $T=0$ is obtained by taking the asymptotic limit $x^{\prime} \rightarrow-\infty$ and $x \rightarrow \infty$ in order to suppress the contribution of the evanescent modes with imaginary values of the wave number, 10 and reinserting $\hbar$;

$$
g=\frac{e^{2}}{\pi \hbar} \sum_{b a}^{\prime} v_{a} v_{b}\left|G_{b a}\left(x, x^{\prime} ; i 0^{+}\right)\right|^{2}
$$


where the primed sum runs over the propagating modes. This expression can be written in terms of the transmission coefficient $t_{a b}$ defined by Eqs. (53) and (55) as

$$
g=\frac{2 e^{2}}{h} \sum_{b a}^{\prime}\left|t_{b a}\right|^{2} .
$$

These are the results obtained with the perturbation theory.

\section{SUMMARY}

We have studied the conductance for the current through a finite central region which is connected to semi-infinite noninteracting leads on the left and the right. Assuming the Coulomb interaction $U$ and the normal scattering potential with time reversal symmetry $V$ can be switched on only for the electrons in the central region, we have stressed the study of the relation between the conductance in the Kubo formalism and the transmission coefficient introduced by making use of the single-particle Green's function. In order to calculate the contributions of the vertex correction for the dc conductance, we have used the diagrammatic analysis for the perturbation expansion with respect to the Coulomb interaction, which was originally applied to the impurity Anderson model for the Kondo alloy. At $T=0$, the contributions of the vertex correction to the dc conductance become zero if the currents are measured in the leads. This is caused by the fact that the asymptotic form of the Green's function in the leads is given by a simple superposition of the incident, transmitted, and reflected waves. Consequently, the conductance is expressed in a Landauer-type form using the transmission coefficient for single-particle-like excitation at the Fermi energy [see Eq. (44)]. At $T=0$, the scattering coefficients are confirmed to satisfy the unitarity condition owing to a Fermi-liquid property $\operatorname{Im} \Sigma\left(x, x^{\prime} ; i 0^{+}\right)=0$, and this property can be derived with the perturbation theory. The results are generalized to a quasi-one-dimensional system with a number of scattering channels [see Eq. (70)]. The analysis used in the present study can also be applied to a tight-binding model, and the similar results can be derived for the model on a lattice. 30 
Throughout the present study, we have assumed that the perturbation expansion in the Coulomb interaction is valid. For instance, in the limit the size of the central region $L \rightarrow 0$, the model is reduced to an Anderson-Wolff impurity and the perturbation theory is valid for all values of $U$. Although it is not evident that the perturbation theory is valid for all values of $U, V$, and $L$ in the general case, the hypothesis may be valid at least for a finite region of the parameter space of the Hamiltonian where $U, V$, and $L$ are small enough.

\section{ACKNOWLEDGMENTS}

We would like to thank H. Ishii for valuable discussions. Allocation of computer time at the Supercomputer Center, Institute for Solid State Physics, University Tokyo, and at the Computer Center of the Institute for Molecular Science, Okazaki National Research Institutes are gratefully acknowledged. This work was partly supported by a Grant-in-Aid from the Ministry of Education, Science, and Culture. 


\section{REFERENCES}

${ }^{1}$ C. W. Beenakker, Phys. Rev. B 44, 1646 (1991).

${ }^{2}$ Y. Meir and N. S. Wingreen, Phys. Rev. Lett. 68, 2512 (1992).

${ }^{3}$ K. Yosida and K. Yamada, Prog. Theor. Phys. 53, 1286 (1975).

${ }^{4}$ K. Yamada, Prog. Theor. Phys. 54, 316 (1975).

${ }^{5}$ H. Shiba, Prog. Theor. Phys. 54, 967 (1975).

${ }^{6}$ A. Yoshimori, Prog. Theor. Phys. 55, 67 (1976).

${ }^{7}$ R. Landauer, Philos. Mag. 21, 863 (1970).

${ }^{8}$ M. Büttiker, Y. Imry, R. Landauer and S. Pinhas, Phys. Rev. B 31, 6207 (1985).

${ }^{9}$ E. N. Economou and C. M. Soukoulis, Phys. Rev. Lett 46, 618 (1981).

${ }^{10}$ D. S. Fisher and P. A. Lee, Phys. Rev. B 23, 6851 (1981).

${ }^{11}$ P. A. Lee and D. S. Fisher, Phys. Rev. Lett. 47, 882 (1981).

12 J. S. Langer and V. Ambegaokar, Phys. Rev. 121, 1090 (1961).

${ }^{13}$ D. L. Maslov and M. Stone, Phys. Rev. B 52, 5539 (1995).

${ }^{14}$ V. V. Ponomarenko, Phys. Rev. B 52, 8666 (1995).

${ }^{15}$ A. Kawabata, J. Phys. Soc. Jpn. 65, 30 (1996).

${ }^{16}$ A. Shimizu, J. Phys. Soc. Jpn. 65, 1162 (1996).

${ }^{17}$ S. Tarucha, T. Honda, and T. Saku, Solid State Commun. 94, 413 (1995).

${ }^{18}$ P. W. Anderson, Phys. Rev. 124, 41 (1961).

${ }^{19}$ P. A. Wolff, Phys. Rev. 124, 1030 (1961).

${ }^{20}$ A. Oguri, Phys. Rev. B 52, 16727 (1995). 
${ }^{21}$ A. Oguri, H. Ishii, and T. Saso, Phys. Rev. B 51, 4715 (1995).

${ }^{22}$ A. Oguri and H. Ishii, Czech. J. Phys. 46, 2411 (1996); J. Magn. \& Magn. Matter. 156, 389 (1996).

${ }^{23}$ S. Hersfield, J. H. Davies, and J. W. Wilkins, Phys. Rev. B 46, 7046 (1992).

${ }^{24}$ W. Izumida, O. Sakai, and Y. Shimizu, J. Phys. Soc. Jpn. 66, 717 (1997).

${ }^{25}$ A. C. Hewson, The Kondo Problem to Heavy Femions (Cambridge University Press, Cambridge, 1993).

${ }^{26}$ A. A. Abrikosov, L. P. Gor'kov, and I. Y. Dzyaloshinskii, Quantum Field Theoretical Methods in Statistical Physics (Pergamon, London, 1965), p. 170.

${ }^{27}$ K. Yamada and K. Yosida, Prog. Theor. Phys. 76, 621 (1986).

${ }^{28}$ G. M. Éliashberg, Zh. Eksp. Teor. Fiz. 41, 1241 (1961) [JETP 14, 886 (1962)].

${ }^{29}$ E. M. Lifshitz and L. P. Pitaevskii, Statistical Physics part 2 (Pergamon, London, 1980), p. 57 .

${ }^{30}$ A. Oguri, unpublished.

${ }^{31}$ C. L. Kane and M. P. A. Fisher, Phys. Rev. Lett. 68, 1220 (1992).

${ }^{32}$ A. Furusaki and N. Nagaosa, Phys. Rev. B 54, R5239 (1992). 


\section{FIGURES}

FIG. 1. Second order skeleton diagrams for the proper self-energy $\Sigma\left(x, x^{\prime} ; i \varepsilon_{n}\right)$.

FIG. 2. Second order skeleton diagrams in terms of the bare vertex function $\gamma_{\sigma \sigma^{\prime}}\left(x_{4}, x_{3} ; x_{2}, x_{1}\right)$.

FIG. 3. Full vertex function $\Gamma_{\sigma_{4} \sigma_{3} ; \sigma_{2} \sigma_{1}}\left(x_{4}, x_{3} ; x_{2}, x_{1}: i \varepsilon_{4}, i \varepsilon_{3} ; i \varepsilon_{2}, i \varepsilon_{1}\right)$.

FIG. 4. Current-current correlation function $K\left(x, x^{\prime} ; i \nu\right)$.

FIG. 5. Diagrams for the $\nu \operatorname{sgn} \nu$ contribution in $K\left(x, x^{\prime} ; i \nu\right)$. The two intermediate Green's functions which yield the singular contribution are marked with the cross.

FIG. 6. Three point vertex function for the current $\Lambda\left(x^{\prime} ; y_{4}, y_{1} ; i \varepsilon+i \nu, i \varepsilon\right)$. 

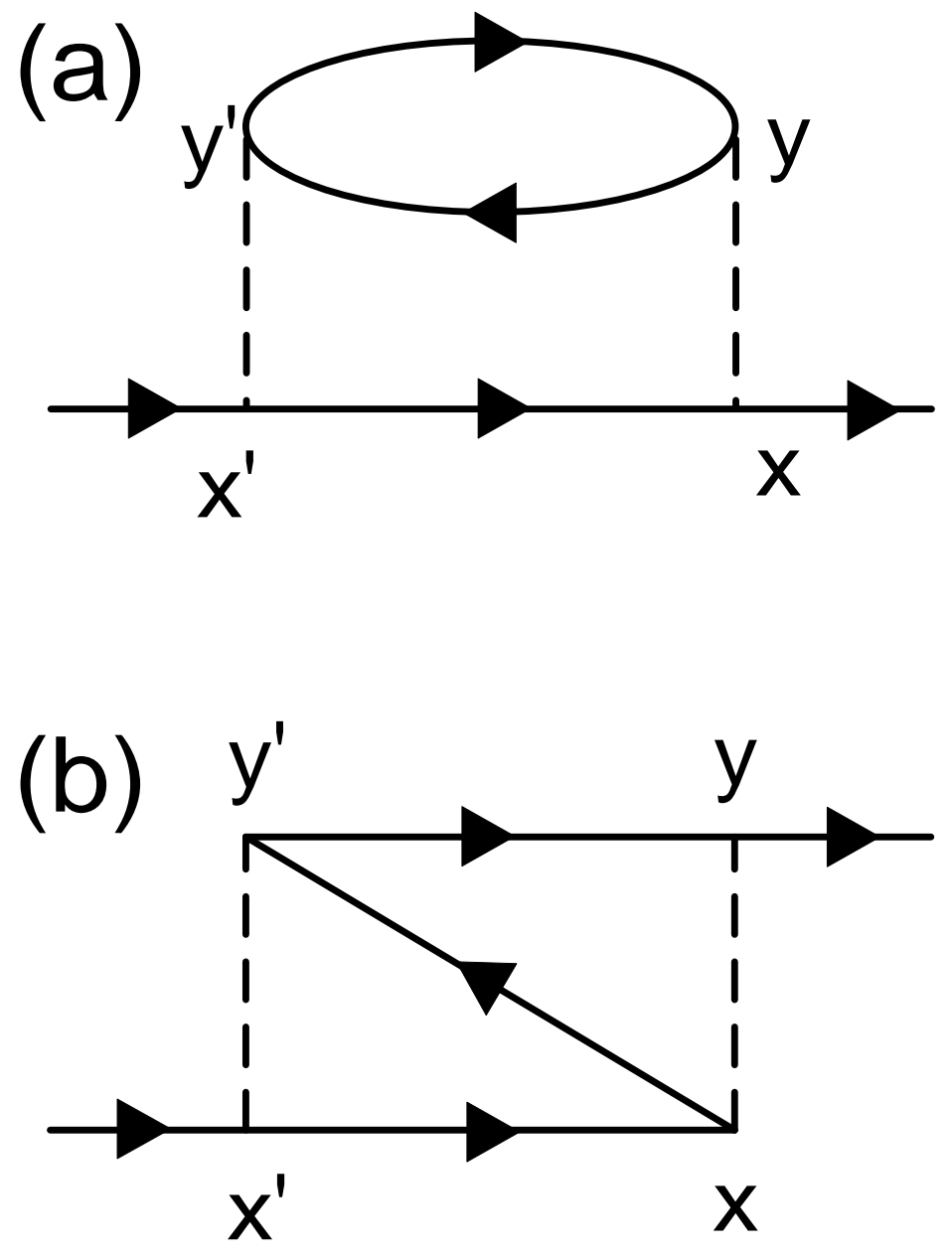

Fig. 1, A. Oguri 


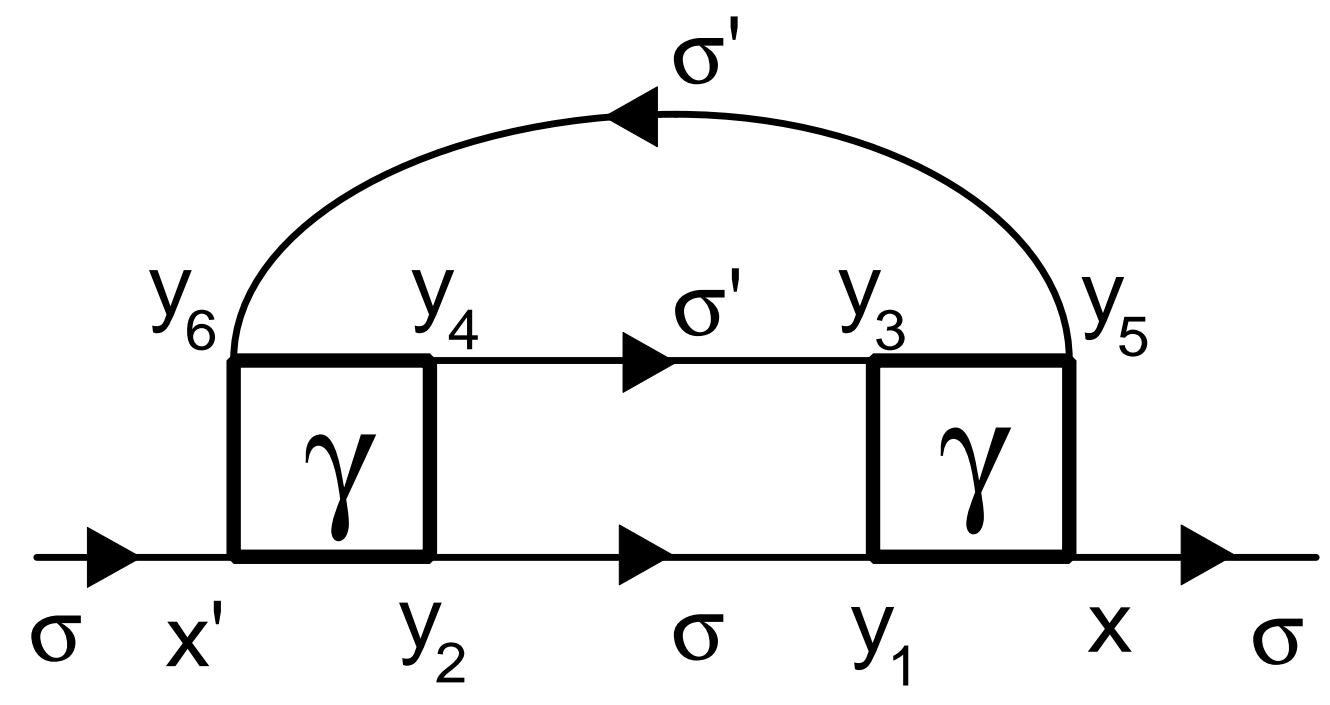

Fig. 2, A. Oguri 


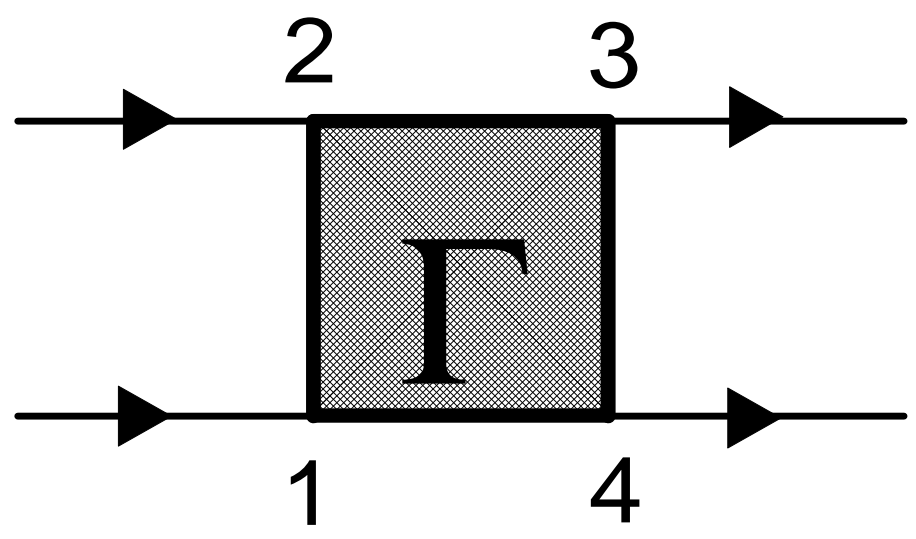

Fig. 3, A. Oguri 

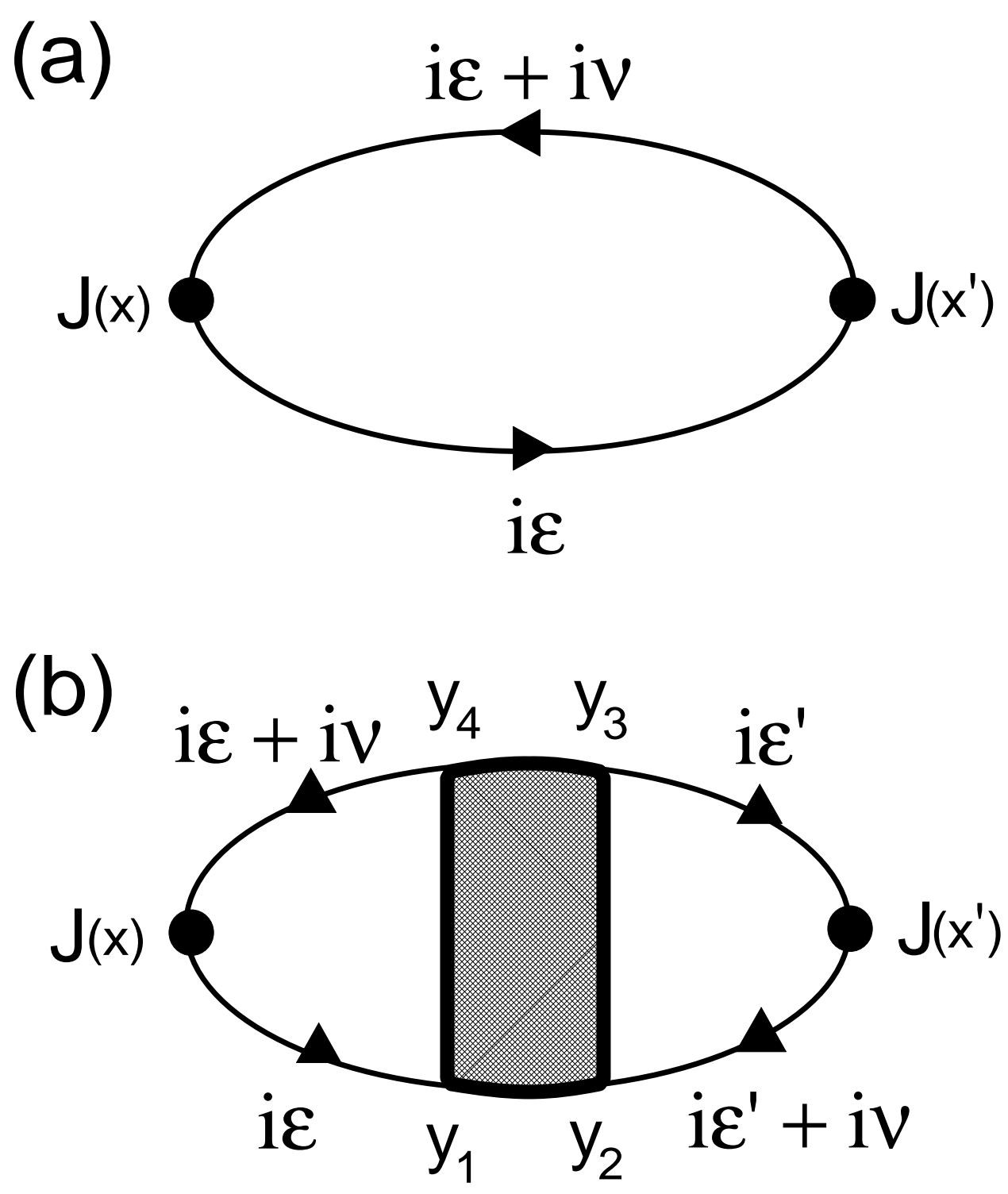

Fig. 4, A. Oguri 


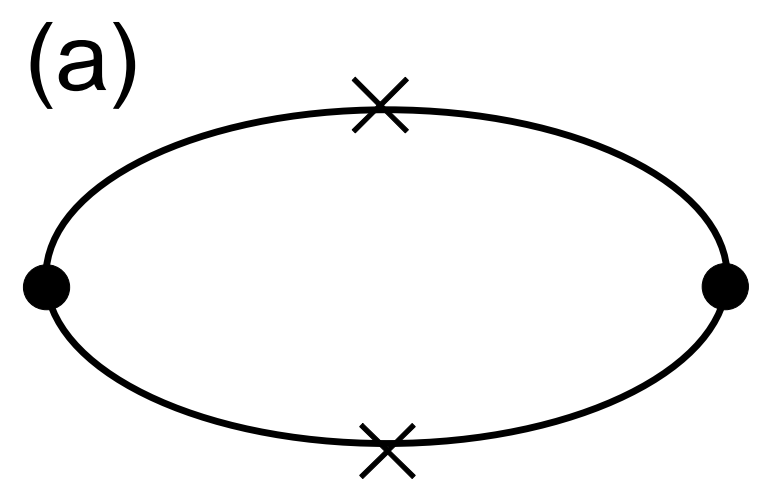

(b1)

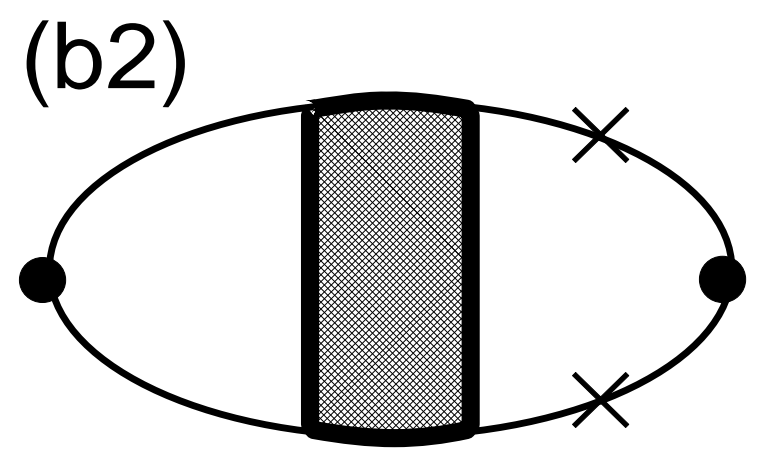

(b3)

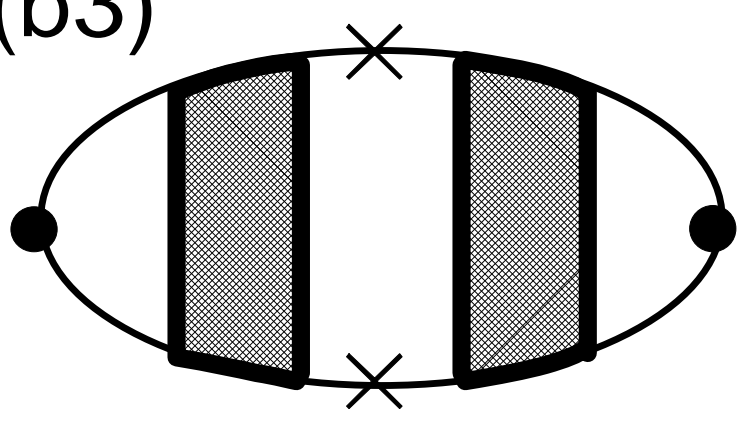

Fig. 5, A. Oguri 


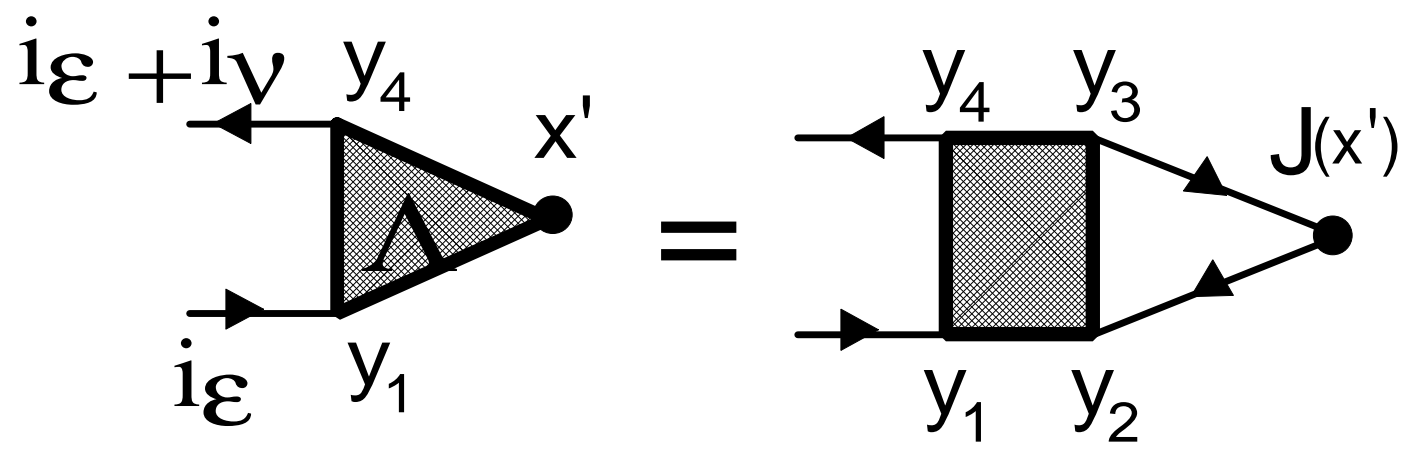

Fig. 6, A. Oguri 